\title{
Getting the balance right
}

DOI:

10.1038/nri2025

URLs

Tcrb http://www.ncbi.nlm.nih. gov/entrez/query.fcgi?db=gen e\&cmd=Retrieve\&dopt=full report\&list_uids $=2157$ Ptcra

http://www.ncbi.nlm.nih.gov/ entrez/query.fcgi?db=gene\&c $\mathrm{md}=$ Retrieve\&dopt=full report\&list_uids $=19208$
What determines the balance between effector and regulatory T-cell development in the thymus? The prevailing view is that agonist selection through the $\mathrm{T}$-cell receptor (TCR) on T-cell progenitors is the determining factor in the development of these cells. However, Hayday and colleagues now show that transconditioning of double-negative (DN; $\left.\mathrm{CD}^{-} \mathrm{CD} 8^{-}\right)$thymocytes by double-positive (DP; $\mathrm{CD} 4^{+} \mathrm{CD} 8^{+}$) thymocytes significantly contributes to the differential competence of these early thymic progenitors to develop into T cells with an effector or regulatory phenotype.

During T-cell development, DN thymocytes can give rise to two

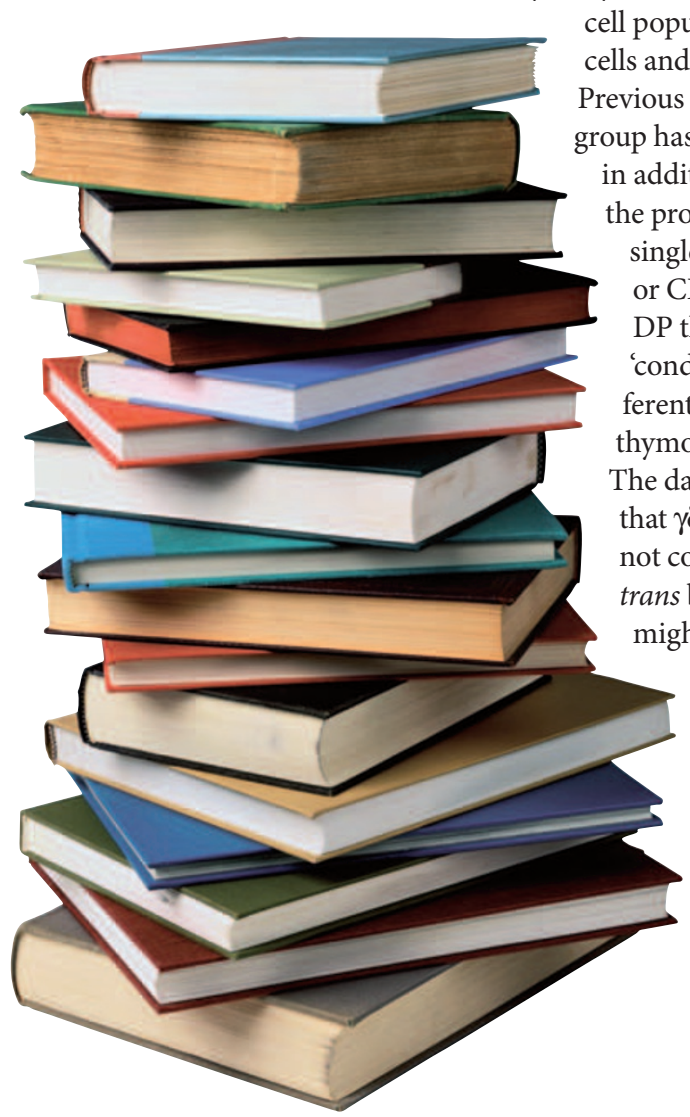
cell populations: $\gamma \delta \mathrm{T}$ cells and DP thymocytes. revious work by this up has shown that, addition to being enitors for single-positive $\mathrm{CD} 4^{+}$ or $\mathrm{CD}^{+} \alpha \beta$ T cells, DP thymocytes also 'condition' the differentiation of DN thymocytes in trans. The data also indicated that $\gamma \delta$ T cells that were not conditioned in trans by DP thymocytes might develop along an alternative pathway.

To further understand this pathway, the authors examined $\gamma \delta$ T-cell development in TCR$\beta$-chain-deficient $\left(\mathrm{Tcrb}^{-/}\right)$mice, which have very few DP thymocytes. $\gamma \delta \mathrm{T}$ cells from $\mathrm{Tcrb}^{-/-}$mice expressed low levels of pro-inflammatory and cytolytic molecules. Furthermore, culture of effector $\mathrm{CD} 4^{+} \mathrm{T}$ cells with activated splenic $\gamma \delta \mathrm{T}$ cells from $\mathrm{Tcrb}^{-/-}$mice, but not wild-type mice, suppressed $\mathrm{CD} 4^{+} \mathrm{T}$-cell expansion in vitro, indicating that these $\gamma \delta \mathrm{T}$ cells might have regulatory potential.

Forkhead box P3 (FOXP3) is a crucial transcription factor in $\mathrm{CD} 4{ }^{+} \mathrm{CD} 25^{\text {hi }}$ regulatory T cells, but its expression by $\gamma \delta \mathrm{T}$ cells has not been reported. In this study a small proportion of $\gamma \delta$ T cells from the spleen or thymus of $\mathrm{Tcrb}^{-/-}$mice, but not wild-type mice, expressed FOXP3. In addition, a fraction of $\gamma \delta \mathrm{T}$ cells from pre-TCR- $\alpha$-chain-deficient $\left(\right.$ Ptcra $\left.^{-/}\right)$ mice expressed FOXP3, highlighting that the differentiation of $\gamma \delta$ T cells is switched towards an aggregate regulatory phenotype in mice with reduced numbers of DP thymocytes.

Although the pre-TCR $\alpha$-chain is absent in $\mathrm{Ptcra}^{-/-}$mice, some $\alpha \beta \mathrm{CD} 4^{+} \mathrm{T}$ cells still develop in these mice. Interestingly, this pool has a higher percentage of CD $25^{\text {hi }}{ }^{2 O X P} 3^{+}$ T cells (25-30\%) than in wild-type mice (2.5-4.5\%), indicating that, in the small population of $\mathrm{CD} 4^{+}$effector $\mathrm{T}$ cells that matures in mice with reduced numbers of DP thymocytes, the ratio of regulatory to effector $\mathrm{T}$ cells is increased.
So how do these studies relate to normal thymocyte differentiation? In wild-type mice, two subpopulations of cells in the DN2 stage of development were identified and referred to as DN2 small (DN2S) and DN2 large (DN2L) cells. The DN2S cells, a product of reduced trans-conditioning, resembled $\mathrm{Ptcra}^{-/-} \mathrm{DN} 2$ cells, and CD4 ${ }^{+} \mathrm{T}$ cells derived from these cells expressed higher levels of a panel of regulatoryT-cell-associated genes, including FOXP3, compared with those derived from the DN2L population.

So, in wild-type mice, trans-conditioning promotes the expansion of DN2L cells that become conventional DP cells, which mature as single positive effector cells or die by negative selection. By contrast, a small population of cells that receive less trans-conditioning (DN2S cells) gain the competence to differentiate into $\mathrm{CD} 25^{\text {hi }} \mathrm{FOXP}^{+}$regulatory $\mathrm{T}$ cells if they encounter cognate self antigen.

Therefore, trans-conditioning by DP cells directly affects the ability of thymocytes to develop into effector or regulatory $\mathrm{T}$ cells. This study might provide insight into the mechanisms of regulatory $\mathrm{T}$-cell enhancement in certain conditions in which DP cells are depleted, such as pregnancy.

Olive Leavy

ORIGINAL RESEARCH PAPER Pennington, D. J. et al. Early events in the thymus affect the balance of effector and regulatory $T$ cells. Nature 444, 1073-1077 (2006) 